\title{
Line and Plane Cover Numbers Revisited ${ }^{\star}$
}

\author{
Therese Biedl ${ }^{1}$, Stefan Felsner ${ }^{20000-0002-6150-1998]}$, Henk Meijer ${ }^{3}$, and \\ Alexander Wolff $4[0000-0001-5872-718 X]$ \\ 1 University of Waterloo, Waterloo, Canada \\ 2 TU Berlin, Berlin, Germany \\ 3 University College Roosevelt, The Netherlands \\ ${ }^{4}$ Universität Würzburg, Würzburg, Germany
}

\begin{abstract}
A measure for the visual complexity of a straight-line crossingfree drawing of a graph is the minimum number of lines needed to cover all vertices. For a given graph $G$, the minimum such number (over all drawings in dimension $d \in\{2,3\}$ ) is called the $d$-dimensional weak line cover number and denoted by $\pi_{d}^{1}(G)$. In $3 \mathrm{D}$, the minimum number of planes needed to cover all vertices of $G$ is denoted by $\pi_{3}^{2}(G)$. When edges are also required to be covered, the corresponding numbers $\rho_{d}^{1}(G)$ and $\rho_{3}^{2}(G)$ are called the (strong) line cover number and the (strong) plane cover number.

Computing any of these cover numbers - except $\pi_{2}^{1}(G)$ - is known to be NP-hard. The complexity of computing $\pi_{2}^{1}(G)$ was posed as an open problem by Chaplick et al. [WADS 2017]. We show that it is NP-hard to decide, for a given planar graph $G$, whether $\pi_{2}^{1}(G)=2$. We further show that the universal stacked triangulation of depth $d, G_{d}$, has $\pi_{2}^{1}\left(G_{d}\right)=$ $d+1$. Concerning $3 \mathrm{D}$, we show that any $n$-vertex graph $G$ with $\rho_{3}^{2}(G)=2$ has at most $5 n-19$ edges, which is tight.
\end{abstract}

\section{Introduction}

Recently, there has been considerable interest in representing graphs with as few objects as possible. The idea behind this objective is to keep the visual complexity of a drawing low for the observer. The types of objects that have been used are straight-line segments $5,5,8,14,15$ and circular arcs $[14,16$.

Chaplick et al. 3 considered covering straight-line drawings of graphs by lines or planes and defined the following new graph parameters. Let $1 \leq l<d$, and let $G$ be a graph. The $l$-dimensional affine cover number of $G$ in $\mathbb{R}^{d}$, denoted by $\rho_{d}^{l}(G)$, is defined as the minimum number of $l$-dimensional planes in $\mathbb{R}^{d}$ such that $G$ has a crossing-free straight-line drawing that is contained in the union of these planes. The weak $l$-dimensional affine cover number of $G$ in $\mathbb{R}^{d}$, denoted by $\pi_{d}^{l}(G)$, is defined similarly to $\rho_{d}^{l}(G)$, but under the weaker restriction that only the vertices are contained in the union of the planes. Clearly, $\pi_{d}^{l}(G) \leq \rho_{d}^{l}(G)$, and if $l^{\prime} \leq l$ and $d^{\prime} \leq d$ then $\pi_{d}^{l}(G) \leq \pi_{d^{\prime}}^{l^{\prime}}(G)$ and $\rho_{d}^{l}(G) \leq \rho_{d^{\prime}}^{l^{\prime}}(G)$. It turns out that it suffices to study the parameters $\rho_{2}^{1}, \rho_{3}^{1}, \rho_{3}^{2}$, and $\pi_{2}^{1}, \pi_{3}^{1}, \pi_{3}^{2}$ :

* S.F. was supported by DFG grant FE 340/11-1, A.W. by DFG grant WO 758/9-1, and T.B. by NSERC. 
Theorem 1 (Collapse of the Affine Hierarchy [3|) For any integers $1 \leq$ $l<3 \leq d$ and for any graph $G$, it holds that $\pi_{d}^{l}(G)=\vec{\pi}_{3}^{l}(G)$ and $\rho_{d}^{l}(G)=\rho_{3}^{l}(G)$.

Disproving a conjecture of Firman et al. [12], Eppstein [10 constructed planar, cubic, 3-connected, bipartite graphs on $n$ vertices with $\pi_{2}^{1}(G) \geq n^{1 / 3}$. Answering a question of Chaplick et al. [3] he also constructed a family of subcubic series-parallel graphs with unbounded $\pi_{2}^{1}$-value. Felsner [11] proved that, for every 4-connected plane triangulation $G$ on $n$ vertices, it holds that $\pi_{2}^{1}(G) \leq \sqrt{2 n}$. Chaplick et al. [4] also investigated the complexity of computing the affine cover numbers. Among others, they showed that in 3D, for $l \in\{1,2\}$, it is NP-complete to decide whether $\pi_{3}^{l}(G) \leq 2$ for a given graph $G$. In $2 \mathrm{D}$, the question has still been open, but a related question was raised by Dujmović et al. 7 already in 2004. They investigated so-called track layouts which are defined as follows. A graph admits a $k$-track layout if its vertices can be partitioned into $k$ ordered independent subsets such that any pair of subsets induces a plane graph (w.r.t. the order of the subsets). The track number of a graph $G, \operatorname{tn}(G)$, is the smallest $k$ such that $G$ admits a $k$-track layout. See also $[6]$ for some recent developments. Note that in general $\pi_{2}^{1}(G) \neq \operatorname{tn}(G)$; for example, $\pi_{2}^{1}\left(K_{4}\right)=2$, whereas $\operatorname{tn}\left(K_{4}\right)=4$. Note further that a 3 -track layout is necessarily plane (which is not the case for $k$-track layouts with $k>3$ ). Dujmović posed the computational complexity of $k$-track layout as an open question.

While it is easy to decide efficiently whether a graph admits a 2-track layout, Bannister et al. 1 answered the open question of Dujmović et al. already for 3 -track layouts in the affirmative. They first showed that a graph has a leveled planar drawing if and only if it is bipartite and has a 3-track layout. Combining this results with the NP-hardness of level planarity, proven by Heath and Rosenberg [13, immediately showed that it is NP-hard to decide whether a given graph has a 3 -track layout. For $k>3$, deciding the existence of a $k$-track layout is NP-hard, too, since it suffices to add to the given graph $k-3$ new vertices each of which is incident to all original vertices of the graph [1].

Our contribution. We investigate several problems concerning the weak line cover number $\pi_{2}^{1}(G)$ and the strong plane cover number $\rho_{3}^{2}(G)$ :

- We settle the open question of Chaplick et al. [4, p. 268] by showing that it is NP-hard to test whether, for a given planar graph $G, \pi_{2}^{1}(G)=2$; see Section 2,

- We show that $G_{d}$, the universal stacked triangulation of depth $d$, (which has treewidth 3) has $\pi_{2}^{1}\left(G_{d}\right)=d+1=\log _{3}\left(2 n_{d}-5\right)+1$, where $n_{d}$ is the number of vertices of $G_{d}$; see Section 3 .

- Eppstein has identified classes of treewidth-2 graphs with unbounded $\pi_{2}^{1}$ value. We give an easy direct argument showing that some 2-tree $H_{d}$ with $n_{d}^{\prime}$ vertices has $\pi_{2}^{1}\left(H_{d}\right) \in \Omega\left(\log n_{d}^{\prime}\right)$; see Appendix B

- Concerning 3D, we show that any $n$-vertex graph $G$ with $\rho_{3}^{2}(G)=2$ has at most $5 n-19$ edges; see Section 4 . This bound is tight. 


\section{Complexity of Computing Weak Line Covers in 2D}

In this section we investigate the computational complexity of deciding whether a graph can be drawn on two lines.

Theorem 2 It is NP-hard to decide whether a given plane (or planar) graph $G$ admits a drawing with $\pi_{2}^{1}(G)=2$.

Proof. Our proof is by reduction from the problem Level Planarity, which Heath and Rosenberg 13 proved to be NP-hard. The problem is defined as follows. A planar graph $G$ is leveled-planar if its vertex set can be partitioned into sets $V_{1}, \ldots, V_{m}$ such that $G$ has a planar straight-line drawing where, for every $i \in\{1, \ldots, m\}$, vertices in $V_{i}$ lie on the vertical line $\ell_{i}: y=i$ and each edge $v_{j} v_{k}$ of $G$ connects two vertices on consecutive lines (that is, $|j-k|=1$ ).

Chaplick et al. 3 have shown that every leveled-planar graph can be drawn on two lines. The converse, however, is not true. For example, $K_{4}$ is not leveledplanar, but $\pi_{2}^{1}\left(K_{4}\right)=2$. Therefore, we modify the given graph in three ways. (a) We replace each edge of $G$ by a $K_{2,4}$-gadget where the two nodes in one set of the bipartition replace the endpoints of the former edge; see Fig. 1a. (b) We add to the graph $G^{\prime}$ that resulted from the previous step a new subgraph $G_{0}$ (two copies of $K_{4}$ sharing exactly two vertices), which we connect by a path to a vertex on the outer face of $G$. (If the outer face is not fixed, we can try each vertex.) In Fig. 1b, $G_{0}$ is yellow and the path is red. The length $L$ of the path is any upper bound on the number of levels of $G^{\prime}$, e.g., the diameter of $G^{\prime}$ (plus 1). (c) We attach to $G_{0}$ a triangulated spiral $S$ (dark green in Fig. 1b). The spiral makes $L+2$ right turns; its final vertex is identified with the outermost vertex of the previous turn. Hence, apart from its many triangular faces, the graph $S+G_{0}$ has a large inner face $F$ of degree $2(L+2)$ and a quadrangular outer face. Let $G^{\prime \prime}$ be the resulting graph. It remains to show that $G$ is leveled-planar if and only if $\pi_{2}^{1}\left(G^{\prime \prime}\right)=2$.

" $\Rightarrow$ ": Fix a leveled-planar drawing of $G$. By doubling the layers and using the new layers to place the large sides of $K_{2,4}$ 's, one easily sees that $G^{\prime}$ is also leveledplanar, see Fig. 1a. As shown in Fig. 1b, the large inner face $F$ of $S+G_{0}$ can be drawn so that it partitions the halflines emanating from the origin into $L$ levels. (It is no problem that consecutive levels are turned by $90^{\circ}$.) Since we chose $L$ large enough (in particular $L \geq 2 m-1$ ), we can easily draw $G^{\prime}$ inside $F$. Note that the red path attached to $G_{0}$ is long enough to reach any vertex on the outer face of $G^{\prime}$. Hence, $\pi_{2}^{1}\left(G^{\prime \prime}\right)=2$.

" $\Leftarrow$ ": Fix a drawing of $G^{\prime \prime}$ on two lines. The two lines cannot be parallel since $G^{\prime \prime}$ contains $K_{2,4}$ and is not outer-planar; so after translation and/or skew we may assume that these two lines are the two coordinate axes. It is not hard to verify that $G_{0}$ must be drawn such that the origin is in its interior, at the common edge of the two $K_{4}$ 's. Furthermore, given this drawing of $G_{0}$, the 3connected spiral $S$ must be drawn as in Fig. 1b. Due to planarity and the fact that $G^{\prime}$ is connected to $G_{0}$ via the red path, $G^{\prime}$ can only be drawn in the interior of $F$. The drawing of $S+G_{0}$ partitions the halflines emanating from the origin 


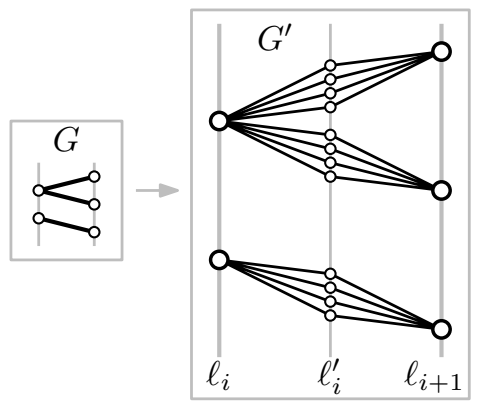

(a) transforming $G$ to $G^{\prime}$

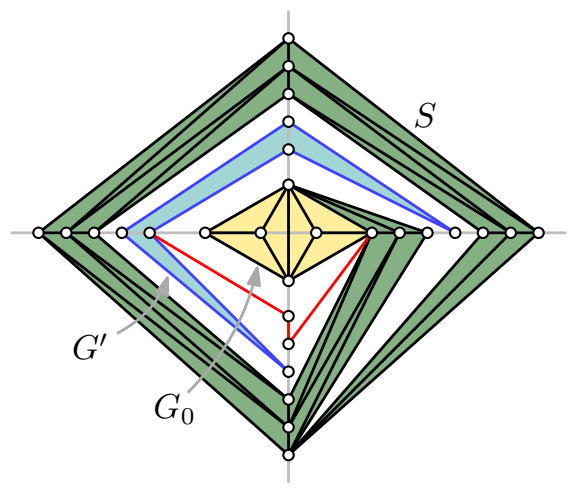

(b) transforming $G^{\prime}$ to $G^{\prime \prime}$

Fig. 1: Our reduction from Level Planarity

into levels, which we number $1,2, \ldots$ starting from the innermost level that contains a vertex of $G^{\prime}$. Inside this face, the only way to draw the $K_{2,4}$-gadgets is as in Fig. 1a, spanning three consecutive levels. This forces all vertices of $G$ to be placed on the odd-numbered levels and the vertices in $G^{\prime}-G$ on the even-numbered levels. Now we can get a level assignment for $G$ by reverting the transformation in Fig. 1a. Hence, $G$ is leveled-planar.

This shows that our reduction is correct. It runs in polynomial time.

\section{Weak Line Covers of Planar 3-Trees in 2D}

In this section we consider the weak line cover number $\pi_{2}^{1}$ for planar graphs, i.e., we are interested in crossing-free straight-line drawings with vertices located on a small collection of lines. Clearly $\pi_{2}^{1}(G)=1$ if and only if $G$ is a forest of paths. The set of graphs with $\pi_{2}^{1}(G)=2$, however, is already surprisingly rich, it contains all trees, outerplanar graphs and subgraphs of grids [1.10].

Stacked triangulations, a.k.a. planar 3-trees or Apollonian networks, are obtained from a triangle by repeatedly selecting a triangular face $T$ and adding a new vertex (the vertex stacked inside $T$ ) inside $T$ with edges to the vertices of $T$. This subdivides $T$ into three smaller triangles, the children of $T$.

For $d \geq 0$ let $G_{d}$ be the universal stacked triangulation of depth $d$, defined as follows. The graph $G_{0}$ is a triangle $T_{0}$, and $G_{d}$ (for $d \geq 1$ ) is obtained from $G_{d-1}$ by adding a stack vertex in each bounded face of $G_{d-1}$. Graph $G_{d}$ has $n_{d}=$ $\frac{1}{2}\left(3^{d}+5\right)$ vertices and $3^{d}$ bounded faces. We show that its weak line cover number is $d+1=\log _{3}\left(2 n_{d}-5\right)+1 \in \Theta\left(\log n_{d}\right)$. (A lower bound of $d$ can also be found in Eppstein's recent book [9, Thm. 16.13].)

Theorem 3 For $d \geq 1$ it holds that $\pi\left(G_{d}\right)=d+1$.

Proof. Here we prove only the lower bound; the construction for the upper bound is illustrated in Fig. 2 and given in Appendix A Let $\mathcal{L}$ be a family of lines covering 


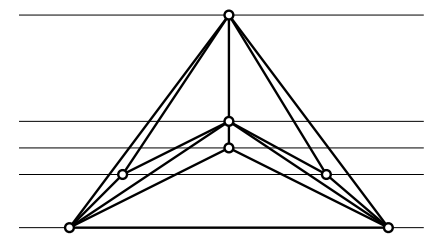

Fig. 2: A drawing of $G_{2}$ that can be extended to a drawing of $G_{3}$ on 5 parallel lines.

the vertices of a drawing of $G_{d}$. Let $a, b$, and $c$ be the vertices of $T_{0}$. We first argue that at least $d$ lines are needed to cover $V \backslash T_{0}$. Let $x_{1}$ be stacked into $T_{0}$. There is a line $L_{1} \in \mathcal{L}$ covering $x_{1}$. Note that $L_{1}$ can intersect only two of the three child triangles of $T_{0}$ (where "intersect" here means "in the interior"). Let $T_{1}$ be a child triangle avoided by $L_{1}$, and let $x_{2}$ be the vertex stacked into $T_{1}$. There is a line $L_{2} \in \mathcal{L}$ covering $x_{2}$. Let $T_{2}$ be a child triangle of $T_{1}$ avoided by $L_{2}$. Iterating this yields $d$ pairwise distinct lines in $\mathcal{L}$.

To find one additional line in $\mathcal{L}$, we distinguish some cases. If a line $L \in \mathcal{L}$ covers two vertices of $T_{0}$, then it covers no inner vertex, and we are done.

Assume some line $L_{a} \in \mathcal{L}$ intersects $x_{1}$ and one vertex of $T_{0}$, say $a$. Let $L_{b}$ and $L_{c}$ be the lines intersecting $b$ and $c$. The lines $L_{a}, L_{b}$, and $L_{c}$ are pairwise different, else we are in the previous case. Of the three child triangles of $T_{0}$, at most one is intersected by $L_{a}$ and at most two each are intersected by $L_{b}$ and $L_{c}$. Therefore, some child triangle $T_{1}$ of $T_{0}$ is intersected by at most one of $L_{a}, L_{b}$, or $L_{c}$. The graph $G_{d-1}$ inside $T_{1}$ requires at least $d-1$ lines for its interior points, and at most one of those lines is $L_{a}, L_{b}$, or $L_{c}$, so in total at least $d+1$ lines are needed.

The argument is similar if no line covers two of $a, b, c$, and $x_{1}$. The four distinct lines supporting $a, b, c$, and $x_{1}$ then intersect at most two child triangles each. So one child triangle $T_{1}$ is intersected by at most two of these lines. Combining the $d-1$ lines needed for the interior of $T_{1}$ with the two lines that do not intersect it, shows that $d+1$ lines are needed.

\section{Maximal Graphs on Two Planes in 3D}

We now switch to dimension $d=3$ and the strong cover number. Obviously any graph $G$ with a drawing that is covered by two planes has at most $6 n-12$ edges since it is the union of two planar graphs. Using maximality arguments and counting, we show that in fact $G$ has at most $5 n-19$ edges if $n \geq 7$. (The restriction $n \geq 7$ is required since for $n=3,4,5,6$ we can have $3,6,9,12$ edges.)

We argue first that our bound is tight. The spine is the intersection of two planes $A$ and $B$. Put a path with $n-4$ vertices on the spine. Add one vertex in each of the four halfplanes and connect each of these vertices to all vertices on the spine and to the vertex on the opposite halfplane; see Fig. 4 in Appendix C. This yields $n-5$ edges on the path and $2(n-4)+1$ edges in each of the two planes, so $5 n-19$ edges in total. 
Theorem 4 Any graph $G$ with $\rho_{3}^{2}(G)=2$ and $n \geq 7$ vertices has at most $5 n-19$ edges.

Proof. Fix a drawing of $G$ on planes $A$ and $B$, inducing planar graphs $G_{A}$ and $G_{B}$ within those planes. Let $G_{A}^{+}$and $G_{B}^{+}$be the graphs obtained from $G_{A}$ and $G_{B}$ by adding any edge that can be inserted without crossing, within the same plane, and with at most one bend on the spine. Clearly it suffices to argue that $G_{A}^{+}$ and $G_{B}^{+}$together have at most $5 n-19$ edges. Let $s$ be the number of vertices on the spine, let $a$ be the number of vertices of $G_{A}^{+}$not on the spine, and let $b$ be the number of vertices of $G_{B}^{+}$not on the spine. Clearly, $a+b+s=n$. We may assume $a \leq b$. We also assume that $1 \leq s \leq n-4$ and that at least one edge of $G_{A}^{+}$crosses the spine (so $2 \leq a \leq b$ ); see Appendix C.

Let $t$ be the number of edges drawn along the spine. These are the only edges that belong to $G_{A}^{+}$and $G_{B}^{+}$. Since $G_{A}^{+}$and $G_{B}^{+}$have at least three vertices each, we can bound the number of edges of $G, m(G)$, as follows:

$$
\begin{aligned}
m(G) & \leq m\left(G_{A}^{+}\right)+m\left(G_{B}^{+}\right)-t \leq 3(s+a)-6+3(s+b)-6-t \\
& =3 n-12+3 s-t \leq 4 n-16+2 s-t .
\end{aligned}
$$

So we must show that $2 s-t \leq n-3$. Let an internal gap be a line segment connecting two consecutive, non-adjacent vertices on the spine. There are $s-t-1$ internal gaps. Let the external gap be the two infinite parts of the spine. Note that at least one edge of $G_{A}^{+}$must cross the external gap, because $G_{A}^{+}$has at least one vertex on each side of the gap, and we could connect the extreme such vertices (or re-route an existing edge) to cross the external gap, perhaps using a bend on the spine. We may further assume that even after such re-routing every internal gap is crossed by at least one edge of $G_{A}^{+}$. Otherwise we could delete all edges of $G_{B}^{+}$passing through the gap, insert the edge between the spine vertices, and re-triangulate the drawing of $G_{B}^{+}$where we removed edges. This would remove an internal gap, but would not decrease the number of edges. Since no edge can cross two gaps, at least $s-t$ edges of $G_{A}^{+}$cross gaps. These edges form a planar bipartite graph with at most $a$ vertices; therefore $s-t \leq 2 a-35^{5}$ This yields $2 s-t \leq s+2 a-3 \leq s+a+b-3=n-3$ as desired.

We conjecture that the following more general statement holds:

Any $n$-vertex graph $G$ with $\rho_{3}^{2}(G)=k$ has at most $(2 k+1)(n-2 k)+k-1$ edges, for all large enough $n$.

Acknowledgments. This research started at the Bertinoro Workshop on Graph Drawing 2017. We thank the organizers and other participants, in particular Will Evans, Sylvain Lazard, Pavel Valtr, Sue Whitesides, and Steve Wismath. We also thank Alex Pilz and Piotr Micek for enlightening conversations.

\footnotetext{
${ }^{5}$ One might be tempted to write a bound of $2 a-4$ here, but we must allow for the possibility of $a=2$, in case of which the planar bipartite graph may have $1=2 a-3$ edges.
} 


\section{References}

1. Bannister, M.J., Devanny, W.E., Dujmović, V., Eppstein, D., Wood, D.R.: Track layouts, layered path decompositions, and leveled planarity. Algorithmica 81(4), 1561-1583 (2019). https://doi.org/10.1007/s00453-018-0487-5

2. Biedl, T., Felsner, S., Meijer, H., Wolff, A.: Line and plane cover numbers revisited. Arxiv report (2019), http://arxiv.org/abs/XXXX.YYYYY

3. Chaplick, S., Fleszar, K., Lipp, F., Ravsky, A., Verbitsky, O., Wolff, A.: Drawing graphs on few lines and few planes. In: Hu, Y., Nöllenburg, M. (eds.) Proc. 24th Int. Symp. Graph Drawing \& Network Vis. (GD'16). LNCS, vol. 9801, pp. 166-180. Springer (2016). https://doi.org/10.1007/978-3-319-50106-2_14, http: //arxiv.org/abs/1607.01196

4. Chaplick, S., Fleszar, K., Lipp, F., Ravsky, A., Verbitsky, O., Wolff, A.: The complexity of drawing graphs on few lines and few planes. In: Ellen, F., Kolokolova, A., Sack, J.R. (eds.) Proc. Algorithms Data Struct. Symp. (WADS'17). LNCS, vol. 10389, pp. 265-276. Springer (2017). https://doi.org/10.1007/978-3-319-621272 23, http://arxiv.org/abs/1607.06444

5. Dujmović, V., Eppstein, D., Suderman, M., Wood, D.R.: Drawings of planar graphs with few slopes and segments. Comput. Geom. Theory Appl. 38(3), 194-212 (2007). https://doi.org/10.1016/j.comgeo.2006.09.002

6. Dujmovic, V., Joret, G., Micek, P., Morin, P., Ueckerdt, T., Wood, D.R.: Planar graphs have bounded queue-number. Arxiv report (2019), http://arxiv.org/abs/ 1904.04791

7. Dujmović, V., Pór, A., Wood, D.R.: Track layouts of graphs. Discrete Math. \& Theor. Comput. Sci. 6(2), 497-522 (2004), https://hal.inria.fr/hal-00959023

8. Durocher, S., Mondal, D.: Drawing plane triangulations with few segments. In: Proc. 26th Canad. Conf. Comput. Geom. (CCCG'14). pp. 40-45 (2014), http: //cccg.ca/proceedings/2014/papers/paper06.pdf

9. Eppstein, D.: Forbidden Configurations in Discrete Geometry. Cambridge Univ. Press (2018)

10. Eppstein, D.: Cubic planar graphs that cannot be drawn on few lines. In: Proc. 35th Int. Symp. Comp. Geom. (SoCG'19). LIPIcs, vol. 129, pp. 32:1-32:15 (2019). https://doi.org/10.4230/LIPIcs.SoCG.2019.32, https://arxiv.org/abs/ 1903.05256

11. Felsner, S.: 4-connected triangulations on few lines. In: Archambault, D., Tóth, C.D. (eds.) Proc. 24th Int. Symp. Graph Drawing \& Network Vis. (GD'16). LNCS, Springer (2019), https://arxiv.org/abs/1908.04524

12. Firman, O., Lipp, F., Straube, L., Wolff, A.: Examining weak line covers with two lines in the plane. In: Biedl, T., Kerren, A. (eds.) Proc. Int. Symp. Graph Drawing Network Vis. (GD'18). LNCS, vol. 11282, pp. 643-645 (2018), https: //link.springer.com/content/pdf/bbm:978-3-030-04414-5/1.pdf, poster

13. Heath, L.S., Rosenberg, A.L.: Laying out graphs using queues. SIAM J. Comput. 21(5), 927-958 (1992). https://doi.org/10.1137/0221055

14. Hültenschmidt, G., Kindermann, P., Meulemans, W., Schulz, A.: Drawing planar graphs with few geometric primitives. J. Graph Alg. Appl. 22(2), 357-387 (2018). https://doi.org/10.7155/jgaa.00473

15. Kindermann, P., Meulemans, W., Schulz, A.: Experimental analysis of the accessibility of drawings with few segments. J. Graph Alg. Appl. 22(3), 501-518 (2018). https://doi.org/10.7155/jgaa.00474

16. Schulz, A.: Drawing graphs with few arcs. J. Graph Alg. Appl. 19(1), 393-412 (2015). https://doi.org/10.7155/jgaa.00366 


\section{Appendix: Missing Proofs}

\section{A Rest of the Proof of Theorem 3}

For the upper bound, we draw $G_{k}$ (for $\left.k=0, \ldots, d\right)$ on $d+2$ layers, i.e., distinct horizontal lines. For every bounded face of $G_{k}$, one edge is short (i.e., either horizontal or connecting two adjacent layers) while the other two edges each cross at least $d-k$ layers in their interior. See also Fig. 2 For $G_{0}$, do this by placing $\left(b_{0}, c_{0}\right)$ horizontally on the lowest layer and $a_{0}$ on the highest layer.

Assume $G_{k}$ (for $0 \leq k<d$ ) has been drawn in this way, and consider a bounded face $T_{k}=\left\{a_{k}, b_{k}, c_{k}\right\}$ of $G_{k}$ into which we want to place the stacked vertex $x_{k}$ to get a drawing of $G_{k+1}$. Say $\left(b_{k}, c_{k}\right)$ is the short edge. Hence the two edges incident to $a_{k}$ cross at least $d-k \geq 1$ layers in their interior. Place $x_{k}$ on the layer adjacent to $a_{k}$ and interior to $T_{k}$ and verify all conditions. For $k=d$ we hence get a drawing of $G_{d}$ on $d+2$ layers. Observe that the top two layers contain only $a_{0}$ and the vertex $x_{0}$ stacked inside $T_{0}$. (This exists by $d \geq 1$.) Hence the line through $a_{0}, x_{0}$, together with the $d$ lines through the other $d$ layers, gives a set of $d+1$ lines supporting the drawing.

\section{B Weak Line Covers of 2-Trees}

We already had the operation of stacking a vertex inside a triangle. We now introduce a similar operation, stacking a vertex onto an edge $(a, b)$, which consists of adding a new vertex $x$ adjacent to $a$ and $b$. Define $H_{0}$ to be the graph consisting of a single edge $(a, b)$, and let $H_{d}$ (for $d \geq 1$ ) be the graph obtained from $H_{d-1}$ by stacking a vertex onto every edge of $H_{d-1}$. The graph $H_{d}$ has $3^{d}$ edges and (since it is a 2 -tree) $n_{d}^{\prime}=\frac{3^{d}+3}{2}$ vertices.

Theorem 1. $\pi_{2}^{1}\left(H_{d}\right) \geq 1+\left\lfloor\frac{d}{8}\right\rfloor \in \Omega\left(\log n_{d}^{\prime}\right)$.

Proof. Fix an arbitrary straight-line planar drawing and line cover of $H_{d+8}$ (for some $d \geq 0$ ); we will show that this line cover needs at least one line more than a line cover of $H_{d}$. The theorem then holds by induction since $H_{0}$ needs one line.

Let $H_{0}=\{(a, b)\}$ be the original edge from which $H_{d+8}$ was built. Let $v_{1}, \ldots, v_{5}$ be the common neighbours of $a$ and $b$ acquired as we extended $H_{0}$ to $H_{5}$ and hence stacked onto $(a, b)$ five times. Let $L$ be the line in the line cover of $H_{d+8}$ that supports $a$. By the pidgeon-hole principle, at least three of $v_{1}, \ldots, v_{5}$ must lie in one (closed) half-space $h$ of $L$; say, $v_{1}, v_{2}$, and $v_{3}$. Sort them such that the rotation at $a$ contains (in counterclockwise order) a ray along $L$, $\left(a, v_{1}\right),\left(a, v_{2}\right),\left(a, v_{3}\right)$, the other ray along $L$ (with the first pair and last pair possibly coinciding). See Fig. 3 .

Let $Q$ be the quadrilateral $\left\langle a, v_{1}, b, v_{3}\right\rangle$. Observe that its sides are edges of $H_{5}$; hence, they have no crossings. When extending $H_{5}$ to $H_{8}$, we stack onto edge $\left(a, v_{2}\right)$ three times; say with vertices $x_{1}, x_{2}$, and $x_{3}$. We now distinguish two cases depending on the location of $b$ : 

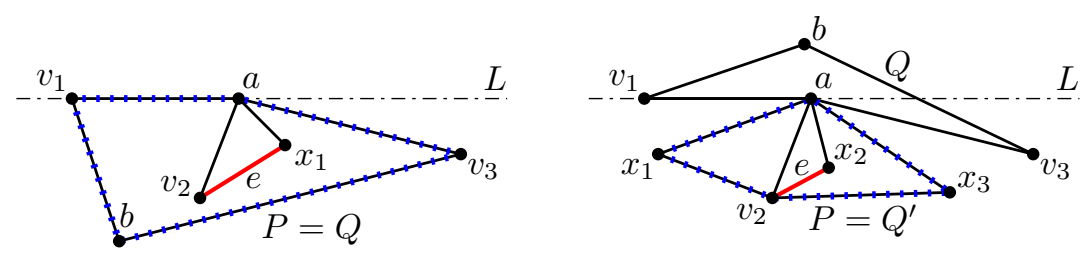

Fig. 3: Finding an edge $e$ (thick red) that is inside polygon $P$ (dotted blue).

Case 1: $b$ is also in $h$. Then $Q$ lies entirely within $h$, and its angle at $a$ is convex or flat. In particular, edge $\left(a, v_{2}\right)$ (which lies between $\left(a, v_{1}\right)$ and $\left.\left(a, v_{3}\right)\right)$ enters the interior of $Q$. By planarity it crosses no edge of $Q$, so $v_{2}$ (and with it also $x_{1}$ ) lie strictly inside $Q$. Set $e=\left(v_{2}, x_{1}\right)$ and $P=Q$.

Case 2: $b$ is not in $h$. Then the angle of $Q$ at $a$ is reflex or flat. This implies that $v_{2}$ (and with it $\left.x_{1}, x_{2}, x_{3}\right)$ lie outside $Q$. Therefore the edges $\left(a, x_{i}\right)$ for $i \in\{1,2,3\}$ must lie between $v_{1}$ and $v_{3}$ in the rotation at $x$; say the rotation is $v_{1}, x_{1}, x_{2}, x_{3}, v_{3}$ (with $v_{2}$ somewhere inbetween). Since we have a straight-line drawing, $x_{1}, x_{2}$, and $x_{3}$ lie in $h$, too. Let $Q^{\prime}$ be the quadrilateral $\left\langle a, x_{1}, v_{2}, x_{3}\right\rangle$. With the same argument as in the previous case (but using $v_{2}$ in place of $b$ ), we see that $x_{2}$ lies strictly within $Q^{\prime}$. Set $e=\left(v_{2}, x_{2}\right)$ and $P=Q^{\prime}$.

In both cases we have found a polygon $P$ such that $L$ does not intersect its interior, and an edge $e$ that lies strictly inside $P$ except perhaps at an endpoint (but that endpoint is not on $L$ ). Edge $e$ has a graph $H_{d}$ stacked onto it, and none of the vertices of this $H_{d}$ (which are either ends of $e$ or strictly inside $P$ ) can be supported by $L$. Hence, a line cover of $H_{d+8}$ must contain at least one line more than a line cover of $H_{d}$.

\section{Missing Cases for the Proof of Theorem 4}

Now we consider the boundary cases.

- If $s=0$ or $a=0$ or $a+b \leq 2$ then $G$ is planar and $m(G) \leq 3 n-6<5 n-19$ (since $n \geq 7$ ). Therefore we may assume $a \geq 1, a+b \geq 3$, and $s=n-a-b \leq$ $n-3$.

- If $s=n-3$ then $a=1$ and $G$ consists of a planar graph in plane $B$ on $n-1$ vertices plus a unique other vertex in $A \backslash B$ adjacent to at most $s$ vertices on the spine. Therefore $m(G) \leq 3(n-1)-6+s \leq 4 n-12 \leq 5 n-19$ by $n \geq 7$. So we may assume $s \leq n-4$, hence $b \geq 3$.

- Assume now that all vertices of $G_{A}^{+}$are to one side of the spine or on the spine. Observe that we may assume $s \geq 3$, for if $s \leq 2$ then, by Equation 1 (which did not use that the vertices of $G_{A}^{+}$occur on both sides), we have $m(G) \leq 3 n-12+3 s \leq 3 n-6<5 n-19$.

Since $s \geq 3$, the convex hull of the drawing of $G_{A}^{+}$contains at least $s+1 \geq$ 4 vertices, hence $m\left(G_{A}^{+}\right) \leq 3(s+a)-7$. This strengthens Equation 1 to 


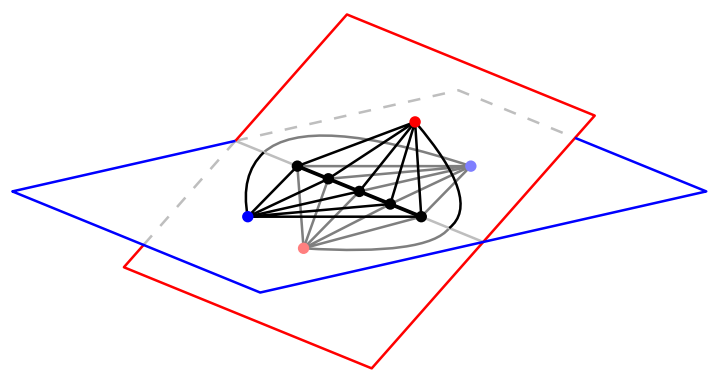

Fig. 4: Example that shows that the bound $5 n-19$ for the number of edges of a 2-plane graph is tight.

$m(G) \leq 4 n-17+2 s-t$, so it suffices to show $2 s-t \leq n-2$. We can therefore afford to have no edge in the external gap. There are no internal gaps (because those could be filled with edges with the same argument as before), so $s-t=1$ and $2 s-t=s+1 \leq n-2$ as desired. 